\title{
Glucocorticoids and Risk of Venous
}

\section{Thromboembolism in Asthma Patients Aged 20-59 Years in the United Kingdom's CPRD 1995-2015}

\author{
Olulade A Ayodele (1D' \\ Howard J Cabral ${ }^{2,3}$ \\ David D McManus ${ }^{4}$ \\ Susan S Jick iD ${ }^{1,5}$ \\ 'Department of Epidemiology, Boston \\ University School of Public Health, \\ Boston, MA, USA; ${ }^{2}$ Department of \\ Biostatistics, Boston University School of \\ Public Health, Boston, MA, USA; \\ ${ }^{3}$ Biostatistics and Research Design \\ Program, Boston University Clinical and \\ Translational Science Institute, Boston, \\ MA, USA; ${ }^{4}$ Department of Medicine, \\ University of Massachusetts Chan \\ Medical School, Boston, MA, USA; \\ ${ }^{5}$ Boston Collaborative Drug Surveillance \\ Program, Lexington, MA, USA
}

Background: Glucocorticoids, the class of steroids used in management of asthma, have been observed to be associated with adverse events such as increased coagulation and inhibition of fibrinolysis. This study evaluated the risk of VTE in relation to the use of glucocorticoids in patients with asthma.

Methods: We conducted a nested case-control study among patients aged 20-59 years with asthma who received at least one glucocorticoid prescription during 1995-2015 in the UKbased Clinical Practice Research Datalink GOLD. We used descriptive analyses and conditional logistic regression to evaluate the risk of VTE associated with glucocorticoid use.

Results: The adjusted ORs (aORs) (95\% CI) for VTE in patients exposed to glucocorticoids were 1.9 (1.6-2.3), $1.4(1.1-1.8)$, and $1.2(0.9-1.5)$ for current, recent, and past glucocorticoid users, respectively, compared to the unexposed. The aORs $(95 \% \mathrm{CI})$ for VTE in patients exposed to systemic glucocorticoid and inhaled glucocorticoids, compared to the unexposed, were $3.5(2.7-4.5)$ and $1.5(1.3-1.8)$, respectively.

Conclusion: Current and systemic glucocorticoid use was associated with a dose-response increased risk of incident idiopathic VTE.

Keywords: glucocorticoids, steroid, VTE, asthma

\section{Introduction}

Asthma, a chronic inflammatory disease of the airways, is a prevalent pulmonary condition, that afflicts close to $7 \%$ of the US population each year. ${ }^{1}$ Inhaled glucocorticoids are widely accepted as standard care for maintaining lung function and controlling asthma symptoms, while oral and intravenous glucocorticoids may be used in acute asthma exacerbation and for severe symptoms.

Glucocorticoids, which have a precursor similar to sex hormones, are the class of steroids prescribed for asthma. Sex hormones are associated with cardiovascular risk factors such as insulin resistance and adverse lipid profiles and are thought to have a complex link with cardiovascular disease. ${ }^{2-5}$ Contrary to studies on testosterone that have shown conflicting results, ${ }^{6}$ glucocorticoids have been observed to increase coagulation and inhibit fibrinolysis. ${ }^{7}$ Therefore, there is biological plausibility for the hypothesis that glucocorticoid use may be associated with an increased risk of venous thromboembolism (VTE) which includes pulmonary embolism (PE) or deep venous thrombosis (DVT). ${ }^{8,9}$ VTE continues to be an important cause of morbidity and mortality. ${ }^{10-12}$ In the United States, over half a million VTE events are treated yearly ${ }^{13}$ with mortality rates as high as $1-6 \%$ for DVT and $12-23 \%$ for PE. ${ }^{14-16}$
Correspondence: Susan S Jick

Boston Collaborative Drug Surveillance Program, II Muzzey Street, Lexington, MA, 0242I, USA

Tel + I 78I 862-6660

Email sjick@bu.edu 
Earlier studies conducted among patients with varied indications for glucocorticoid use (including asthma, joint disorders, connective tissue disorders, inflammatory bowel disease, upper and lower respiratory disorders) compared to non-users have shown increases in the risk of VTE., ${ }^{7,117-22}$ However, it is challenging to tease apart the prothrombotic effect of glucocorticoids from the inflammatory and hypercoagulable state induced either chronically by the disease state or acutely with episodes of asthma exacerbation. In this study, we used a population-based nested case-control study in the United Kingdom Clinical Practice Research Datalink (CPRD) GOLD to evaluate the association between glucocorticoid use in patients with asthma and the risk of developing VTE.

\section{Methods}

CPRD GOLD is an ongoing longitudinal database, established in 1987, of over 400 general practices in the UK. It contains data on a representative sample of more than 10 million UK patients recorded by general practitioners (GPs) using standard software and coding systems. In addition to patient demographic information, CPRD GOLD includes information on medical diagnoses, prescriptions issued in primary care (including dosage and quantity), hospitalizations, referrals, deaths, laboratory tests, and lifestyle habits, such as smoking and alcohol use. Large validation studies have demonstrated the accuracy and completeness of data captured in CPRD GOLD for use in pharmacoepidemiological research. ${ }^{14,22,23}$

The study population was comprised of asthma patients who had at least one glucocorticoid prescription between January 1, 1995, and December 31, 2015, in CPRD GOLD and who were aged 20-59 years at the first glucocorticoid prescription. Patients were required to have at least 1 year of recorded history in the database before their study entry date (later of date of the first asthma diagnosis or first glucocorticoid prescription). We then excluded all patients who had any diagnosis of non-melanoma skin cancer, or strong cardiovascular risk factors ${ }^{24,25}$ (defined as patients with a history of VTE, cerebrovascular disease, coronary heart disease, heart failure, coagulopathies, vasculitis, and chronic kidney disease) before the study entry date, to include only relatively healthy patients in the study population.

\section{Outcome}

The study outcome was incident (first ever code for VTE), idiopathic VTE (DVT or PE) that occurred after study entry. Only idiopathic VTE cases, those that did not have a code for another proximate cause of VTE (including pregnancy, immobility, prolonged hospitalization, fractures, multiple traumas, orthopedic surgery involving long bones or pelvis, and other major surgery) recorded in CPRD GOLD within 90 days prior to the VTE diagnosis date, were included. Patients were required to have one or more codes for anticoagulants (including unfractionated heparin, low-molecular-weight heparins (LMWH) and fondaparinux) present after the VTE diagnosis to be considered a case. This requirement was implemented to increase the specificity of our case definition.

The index date was defined as the date of the first VTE diagnosis in the cases or the matched date for controls.

\section{Control Selection}

From the base population, we matched up to four controls to each case on index date (same date as their matched case), age within 3 years, sex, general practice and the patient's record start date in CPRD GOLD \pm 3 years. Where insufficient numbers of controls were found, we removed the requirement to match on GP practice. The same exclusion criteria used in cases were applied to controls.

\section{Exposure}

Glucocorticoid use was derived from medication codes available in CPRD GOLD. We evaluated exposure in three different ways to assess the timing of glucocorticoid exposure and risk of VTE. We defined exposure as any glucocorticoid prescription whose filled use ended within 1 year before the index date (where use was defined as the prescription length +30 days). Glucocorticoid use that ended more than 1 year before the index date was considered as unexposed.

We also categorized glucocorticoid use according to the timing of the most recent glucocorticoid prescription received prior to the index date. Current use was defined as any glucocorticoid use that continued to the index date. Recent use was defined as any glucocorticoid use that ended less than 180 days prior to the index date, and not including the index date. Past use was defined as any glucocorticoid use that ended from 180 days to less than 1 year prior to the index date. Unexposed was defined as glucocorticoid use that ended 1 year or more prior to the index date.

We further categorized exposure by route of administration: systemic use comprising oral and injectable 
[intramuscular (IM) or intravenous (IV)] glucocorticoids, and inhalational use comprising inhaled, intranasal, and nebulized glucocorticoids. There were only 45 records of IM or IV glucocorticoid use $(0.8 \%)$ in this population; therefore, we were unable to assess IM/IV glucocorticoid use independently.

We also evaluated duration of glucocorticoid use as the number of prescriptions recorded any time before the index date $(1-6,7-24,25-49,50+)$. We evaluated the total cumulative dose of glucocorticoids (sum of number of pills in each prescription times prescription strength for oral glucocorticoids; sum of number of inhalers times dose for inhaled use; and number of injections times strength for injectable glucocorticoids) recorded before the index date (1-999; $1000-9999 ; 10,000+\mu \mathrm{g})$. We further subcategorized the number and dose of glucocorticoid prescription by the recency of exposure prior to the index date, ie, current, recent, or past.

\section{Covariates}

We controlled for confounding by age, sex, calendar time, and general practice attended through matching. We evaluated additional covariates as potential confounders, including age at first glucocorticoid prescription, smoking (current, past, never smoker or unknown, BMI $(<25,25-$ $<30, \geq 30 \mathrm{~kg} / \mathrm{m}^{2}$ and unknown), oral contraceptive or hormone replacement use, and comorbidities (including history of phlebitis, COPD, epilepsy, alcohol use, diabetes, and hypertension). A patient was considered to have a comorbidity if there was at least one Read code for the condition in their record prior to the index date.

\section{Data Analysis}

We estimated crude and adjusted ORs and 95\% CI of the association of glucocorticoid use compared to no glucocorticoid exposure using conditional logistic regression modelling for matched pairs using PROC LOGISTIC. In addition to the matching factors, we also evaluated variables that were structurally deemed to be potential confounders. A covariate was included in the model if it changed the main effect measure by at least $10 \%$.

We conducted stratified analyses to examine effect measure modification (EMM) by sex, age group, and obesity status.

Separate analyses were also conducted to examine the independent association of recency of glucocorticoid use and route of glucocorticoid administration with VTE. In both cases, the unexposed group served as the reference category.

We conducted sensitivity analyses to test whether the effect estimate would change materially if we limited the analysis to cases who did not have a hormone replacement therapy or oral contraceptive pill prescription recorded in the 90 days before the index date $(n=5335)$. In separate sensitivity analyses, we restricted to patients who were matched perfectly to controls, that is, those that could be matched to controls within the same GP practice. Analyses were conducted using SAS statistical software (version 9.4, SAS Institute).

\section{Data Use and Ethical Approval}

This study is based in part on data from the Clinical Practice Research Datalink obtained under license from the UK Medicines and Healthcare Products Regulatory Agency. The data is provided by patients and collected by the NHS as part of their care and support. This study was approved by the Independent Scientific Advisory Committee (ISAC) for Medicines and Healthcare Products Regulatory Agency database research (protocol no: 17 138R), and the protocol was made available to the journal reviewers upon request. All data accessed complied with relevant data protection and privacy regulations.

\section{Results}

We identified 3336 VTE cases in the population of 437,842 asthma patients aged 20-59 years who met the study inclusion criteria. After applying our exclusion criteria, 1168 idiopathic VTE cases remained to which we matched 4672 controls.

The distribution of potential VTE risk factors and patient characteristics among cases and controls as well as bivariate ORs and associated 95\% CIs are presented in Table 1. The distribution of most risk factors was similar between cases and controls. However, cases were more likely than controls to have a history of diabetes, phlebitis, COPD, smoking and a higher BMI $\left(>30 \mathrm{~kg} / \mathrm{m}^{2}\right)$. Women with VTE were more likely to have received hormone replacement therapy or an oral contraceptive prescription than controls. Twenty-five percent of cases had not received a glucocorticoid prescription in the year prior to their VTE diagnosis compared to $38 \%$ among matched controls. Fifty-seven percent, 10\%, and $7 \%$ of cases were current, recent, and past glucocorticoid users respectively compared to $43 \%, 11 \%$, and $9 \%$ of 
Table I Characteristics and Bivariate Odds Ratios in Venous Thromboembolism (VTE) Cases and Matched Controls: Asthma Patients Aged 20-59 Years in CPRD GOLD 1995-2015

\begin{tabular}{|c|c|c|c|c|}
\hline & Cases $(n=1 \mid 68)$ & Controls $(n=4672)$ & OR & $95 \% \mathrm{Cl}$ \\
\hline \multicolumn{5}{|c|}{ Age at Index Date (years) } \\
\hline 20-29 & $86(7.4)$ & $344(7.4)$ & $N A^{a}$ & $N A^{a}$ \\
\hline $30-39$ & $207(17.7)$ & $828(17.7)$ & $N A^{a}$ & $N A^{a}$ \\
\hline $40-49$ & $284(24.3)$ & II $36(24.3)$ & $N A^{a}$ & $N A^{a}$ \\
\hline 50-59 & $591(50.6)$ & $2364(50.6)$ & $N A^{a}$ & $N A^{a}$ \\
\hline \multicolumn{5}{|l|}{ Sex } \\
\hline Male & $483(4 I .4 \%)$ & $1932(41.4 \%)$ & $N A^{a}$ & $N A^{a}$ \\
\hline Female & $685(58.7 \%)$ & $2740(58.7 \%)$ & $N A^{a}$ & $N A^{a}$ \\
\hline \multicolumn{5}{|l|}{ BMI $\left(\mathbf{k g} / \mathrm{m}^{2}\right)$} \\
\hline$<18$ & II (0.9\%) & $48(1.0 \%)$ & 1.4 & $0.7-2.8$ \\
\hline 18-24 & 241 (20.6\%) & 1526 (32.7\%) & I.0 (reference) & I.0 (reference) \\
\hline $25-30$ & $289(24.7 \%)$ & I 357 (29.1\%) & 1.4 & I.I-I.7 \\
\hline$>30$ & $532(45.6 \%)$ & 1284 (27.5\%) & 2.7 & $2.3-3.2$ \\
\hline Unknown & $95(8.1 \%)$ & 457 (9.8\%) & 1.3 & I.0-1.7 \\
\hline \multicolumn{5}{|l|}{ Smoking Status } \\
\hline Non-smoker & 490 (42.0\%) & $2219(47.5)$ & I.0 (reference) & 1.0 (reference) \\
\hline Current smoker & 286 (24.5\%) & $989(21.2)$ & 1.3 & $1.1-1.5$ \\
\hline Past-smoker & 361 (30.9\%) & 1342 (28.7) & 1.2 & I.0-1.4 \\
\hline Unknown & 31 (2.7\%) & $122(2.6)$ & I.I & $0.7-1.8$ \\
\hline \multicolumn{5}{|l|}{ Comorbidities } \\
\hline Phlebitis & 101 (8.7\%) & $66(1.4)$ & 6.8 & $4.9-9.4$ \\
\hline Hypertension & $196(16.8 \%)$ & $842(18.0)$ & 0.9 & $0.8-1.1$ \\
\hline Diabetes & 69 (5.9\%) & $189(4.1)$ & 1.5 & I.I-2.0 \\
\hline Hyperlipidemia & 96 (8.2\%) & 495 (I0.6\%) & 0.7 & $0.6-0.9$ \\
\hline Alcohol abuse & $14(1.2 \%)$ & $48(1.0 \%)$ & 1.2 & $0.6-2.1$ \\
\hline COPD & 114 (9.8\%) & 327 (7.0\%) & 1.5 & $1.2-1.9$ \\
\hline Epilepsy & $6(0.5 \%)$ & $15(0.3 \%)$ & 1.6 & $0.6-4.1$ \\
\hline \multicolumn{5}{|c|}{ Other Proximate Causes of VTE within 90 Days } \\
\hline HRT or OC use $\mathrm{b}^{\mathrm{b}}$ & $123(10.5 \%)$ & 382 (8.2\%) & 1.4 & I.I-I.7 \\
\hline
\end{tabular}

Notes: a Matching factor; ${ }^{\mathrm{b}} \mathrm{Among}$ women only.

Abbreviations: OR, odds ratio; $95 \% \mathrm{Cl}, 95 \%$ confidence interval; NA, not applicable; HRT, hormone replacement therapy; OC, oral contraceptive.

controls. A high proportion of cases (15\%) had systemic (injectable or oral) glucocorticoid use in the year prior to the index date compared to $6 \%$ in controls.
Table 2 shows the distribution of patient characteristics and potential confounders by glucocorticoid exposure status among controls. Glucocorticoid exposed patients were 
Table 2 Characteristics of Exposed and Unexposed Controls at Index Date: Asthma Patients Aged 20-59 Years in CPRD GOLD 1995-2015

\begin{tabular}{|c|c|c|c|}
\hline & Exposed $(n=2909)$ & Unexposed $(n=\mid 763)$ & OR $(95 \% \mathrm{Cl})$ \\
\hline \multicolumn{4}{|l|}{ Age at Index (years) } \\
\hline 20-29 & $176(6.1 \%)$ & $168(9.5 \%)$ & I.0 (reference) \\
\hline $30-39$ & $392(13.5 \%)$ & $436(24.7 \%)$ & $0.9(0.7-I . I)$ \\
\hline $40-49$ & $665(22.9 \%)$ & 47। (26.7\%) & $1.3(1.1-1.7)$ \\
\hline $50-59$ & $1676(57.6 \%)$ & $688(39.0 \%)$ & $2.3(1.8-2.9)$ \\
\hline \multicolumn{4}{|l|}{ Sex } \\
\hline Male & $1213(41.7 \%)$ & 719 (40.8\%) & I.0 (reference) \\
\hline Female & 1696 (58.3\%) & 1044 (59.2\%) & $1.0(0.9-1.1)$ \\
\hline \multicolumn{4}{|l|}{ BMI $\left(\mathrm{kg} / \mathrm{m}^{2}\right)$} \\
\hline$<18$ & $30(1.0 \%)$ & $18(1.0 \%)$ & I.I (0.6-I.9) \\
\hline $18-24$ & $928(31.9 \%)$ & $598(33.9 \%)$ & I.0 (reference) \\
\hline $25-30$ & 859 (29.5\%) & $498(28.3 \%)$ & I.I (I.0-1.3) \\
\hline$>30$ & $852(29.3 \%)$ & $432(24.5 \%)$ & $1.3(1.1-1.5)$ \\
\hline Unknown & $240(8.3 \%)$ & $217(12.3 \%)$ & $0.7(0.6-0.9)$ \\
\hline \multicolumn{4}{|l|}{ Smoking Status } \\
\hline Non-smoker & $|35|(46.4 \%)$ & $868(49.2)$ & I.0 (reference) \\
\hline Current smoker & $596(20.5 \%)$ & $393(22.3)$ & $1.0(0.8-1.1)$ \\
\hline Past-smoker & 898 (30.9\%) & $444(25.2)$ & $1.3(1.1-1.5)$ \\
\hline Unknown & $64(2.2 \%)$ & $58(3.3)$ & $0.7(0.5-1.0)$ \\
\hline \multicolumn{4}{|l|}{ Comorbidities } \\
\hline Phlebitis & $51(1.8 \%)$ & 15 (0.9\%) & $2.1(1.2-3.7)$ \\
\hline Drug abuse & $10(0.3 \%)$ & $9(0.5 \%)$ & $0.7(0.3-1.7)$ \\
\hline Hypertension & $611(21.0 \%)$ & $231(13.1 \%)$ & $1.8(1.5-2.1)$ \\
\hline Diabetes & I 38 (4.7\%) & 51 (2.9\%) & $1.7(1.2-2.3)$ \\
\hline Hyperlipidemia & 345 (11.9\%) & $150(8.5 \%)$ & $1.4(1.1-1.8)$ \\
\hline Alcohol abuse & $33(1.1 \%)$ & $15(0.9 \%)$ & $1.3(0.7-2.5)$ \\
\hline COPD & $293(10.1 \%)$ & $34(1.9)$ & $5.7(4.0-8.2)$ \\
\hline Epilepsy & $12(0.4 \%)$ & NR & $2.4(0.7-8.6)$ \\
\hline \multicolumn{4}{|l|}{ HRT or OCP Use } \\
\hline$<90$ days before index & $253(8.7 \%)$ & $129(7.3 \%)$ & $1.2(1.0-1.5)$ \\
\hline
\end{tabular}

Notes: Categorical variables are presented as N (\%). Exposed = glucocorticoid use less than I year prior to the index date; Unexposed = glucocorticoid use I year or more prior to the index date; Current use = glucocorticoid use that continued to the index date; Recent use $=$ glucocorticoid use less than 180 days prior to the index date; Past use= glucocorticoid use $\mathrm{I} 80$ days-less than I year prior to the index date.

Abbreviations: OR, odds ratio; $95 \% \mathrm{Cl}$, 95\% confidence interval; HRT, hormone replacement therapy; OCP, oral contraceptive pills; NA, not applicable; NR, not reportable. 
Table 3 The Association Between Any Glucocorticoid Exposure and VTE for Asthma Patients Aged 20-59 Years in CPRD I995-20I5 $(n=5840)$

\begin{tabular}{|c|c|c|c|c|}
\hline \multicolumn{5}{|c|}{ Odds Ratio (95\% Confidence Interval) for Venous Thromboembolism (VTE) } \\
\hline & Cases/Controls & Model I ${ }^{\mathrm{a}}$ OR $(95 \% \mathrm{Cl})$ & Model $2^{b}$ OR $(95 \% \mathrm{Cl})$ & Model $3^{c}$ OR $(95 \% \mathrm{Cl})$ \\
\hline \multicolumn{5}{|c|}{ Exposure Status } \\
\hline Unexposed & $296 / 1763$ & 1.0 (reference) & 1.0 (reference) & 1.0 (reference) \\
\hline Exposed & $872 / 2909$ & $1.8(1.6-2.1)$ & $1.7(1.5-2.0)$ & $1.7(1.5-2.0)$ \\
\hline \multicolumn{5}{|l|}{ Recency } \\
\hline Unexposed & $296 / 1763$ & 1.0 (reference) & 1.0 (reference) & 1.0 (reference) \\
\hline Current & $670 / 1992$ & $2.1(1.8-2.5)$ & $1.9(1.6-2.3)$ & $1.9(1.6-2.3)$ \\
\hline Recent & $118 / 495$ & $1.4(1.1-1.8)$ & $1.4(1.1-1.8)$ & $1.4(1.1-1.8)$ \\
\hline Past & $84 / 422$ & $1.2(0.9-1.6)$ & $1.2(0.9-1.6)$ & $1.2(0.9-1.5)$ \\
\hline \multicolumn{5}{|l|}{$\begin{array}{l}\text { Route of } \\
\text { Exposure }\end{array}$} \\
\hline Unexposed & $296 / 1763$ & I.0 (reference) & I.0 (reference) & I.0 (reference) \\
\hline Systemic & $|7| / 28 \mid$ & $3.8(3.0-4.8)$ & $3.5(2.7-4.5)$ & $3.5(2.7-4.5)$ \\
\hline Inhaled & $701 / 2628$ & $1.6(1.4-1.9)$ & $1.5(1.3-1.8)$ & $1.5(1.3-1.8)$ \\
\hline
\end{tabular}

Notes: anadjusted. 'bdjusted for phlebitis, COPD, diabetes, BMI, smoking. Conditional on the matching factors. 'Adjusted for phlebitis, COPD, diabetes, BMI, smoking, hormone replacement/OCP, epilepsy, alcohol. Conditional on the matching factors. Exposed = glucocorticoid use less than I year prior to the index date. Unexposed = glucocorticoid use I year or more prior to the index date; Current use = glucocorticoid use less than 90 days prior to the index date; Recent use $=$ glucocorticoid use 90 days-less than 180 days prior to the index date; Past use $=$ glucocorticoid use 180 days-less than 1 year prior to the index date.

Abbreviations: OR, odds ratio; $95 \% \mathrm{Cl}, 95 \%$ confidence interval.

older, more likely to be obese, have a history of phlebitis, diabetes, hypertension and COPD than unexposed patients.

We observed a twofold increase in the risk of VTE among current glucocorticoid users $(\mathrm{aOR}=1.9,95 \% \mathrm{CI}$ : 1.6-2.3), a minimal increase in risk for recent glucocorticoid users $(\mathrm{aOR}=1.4,95 \% \mathrm{CI}: 1.1-1.8)$, and a near null effect for past glucocorticoid users $(\mathrm{aOR}=1.2,95 \% \mathrm{CI}$ : 0.9-1.5) compared to the unexposed. See Table 3. We observed the highest risk of VTE among current and recent glucocorticoid users with fewer glucocorticoid prescriptions and lower cumulative dose. Patients who had current exposure with 1-6 prescriptions or less than 1000 $\mu \mathrm{g}$ of cumulative glucocorticoid dose had a threefold higher risk of VTE compared to unexposed patients. In contrast, patients with $25+$ prescriptions or over 10,000 $\mu \mathrm{g}$ of past use had no increased risk of VTE when compared to the unexposed, suggesting an acute effect of glucocorticoids on the risk of VTE. See Table 4.

When we examined the route of glucocorticoid administration, we observed a higher risk among those exposed to a systemic glucocorticoid $(\mathrm{aOR}=3.5,95 \% \mathrm{CI}: 2.7-4.5)$ than among those exposed to an inhaled glucocorticoid (aOR=1.5, 95\% CI: 1.3-1.8) compared to the unexposed, suggesting a dose-response relationship between glucocorticoid use and risk of idiopathic VTE. See Table 5.

When we examined the recency and route of administration (Table 5), we observed an effect primarily in current users and in those with systemic glucocorticoid use $(\mathrm{aOR}=4.8,95 \% \mathrm{CI}: 3.7-6.4)$; further supporting a doseresponse effect.

$\underline{\text { Suppl } 1}$ shows the results stratified by sex. The effect was in the same direction for males and females, but the magnitude of effects differed in models where we examined recency of administration (female current glucocorticoid use: $\mathrm{aOR}=2.3,95 \%$ CI:1.9-2.9; male current glucocorticoid use: $\mathrm{aOR}=1.5,95 \% \mathrm{CI}: 1.2-2.0$ ), and route of administration (female systemic glucocorticoid use: $\mathrm{aOR}=3.9,95 \% \mathrm{CI}: 2.9-5.3$; male systemic glucocorticoid use: $a \mathrm{OR}=2.995 \% \mathrm{CI}: 1.9-4.4)$. This was not explained by hormone replacement therapy or oral contraceptive pill use. When we stratified the analyses by age, the aORs were slightly higher in patients aged 20-39 
Table 4 Crude and Adjusted Odds Ratios for the Association Between Glucocorticoid Exposure and Venous Thromboembolism by Timing of Use and Number of Prescriptions Received: Asthma Patients Aged 20-59 Years in CPRD GOLD I995-20I5

\begin{tabular}{|c|c|c|c|c|}
\hline \multicolumn{5}{|c|}{ Odds Ratio (95\% Confidence Interval) for Venous Thromboembolism (VTE) } \\
\hline & Cases/Controls (1 I68/4672) & Model I ${ }^{\mathrm{a}}$ OR $(95 \% \mathrm{Cl})$ & Model $2^{\text {b }}$ OR $(95 \% \mathrm{Cl})$ & Model 3c OR (95\% Cl) \\
\hline \multicolumn{5}{|c|}{ Recency and Number of Glucocorticoid Prescriptions } \\
\hline Unexposed & $296 / 1763$ & 1.0 (reference) & 1.0 (reference) & 1.0 (reference) \\
\hline Current use & $670 / 1992$ & $2.1(1.8-2.5)$ & $1.9(1.6-2.3)$ & $1.9(1.6-2.3)$ \\
\hline $1-6$ & $133 / 297$ & $2.8(2.1-3.6)$ & $2.9(2.2-3.8)$ & $2.9(2.2-3.7)$ \\
\hline 7-24 & $195 / 585$ & $2.1(1.7-2.5)$ & $1.9(1.6-2.4)$ & $1.9(1.6-2.4)$ \\
\hline $25-49$ & $152 / 455$ & $2.1(1.7-2.6)$ & $1.8(1.4-2.2)$ & $1.8(1.4-2.2)$ \\
\hline $50+$ & $190 / 655$ & $1.8(1.4-2.2)$ & $1.6(1.2-2.0)$ & $1.6(1.2-2.0)$ \\
\hline Recent use & $118 / 495$ & $1.4(1.1-1.8)$ & $1.4(1.1-1.8)$ & $1.4(1.1-1.8)$ \\
\hline $1-6$ & $38 / 148$ & $1.5(1.1-2.3)$ & $1.6(1.1-2.4)$ & $1.6(1.1-2.4)$ \\
\hline $7-24$ & $48 / 201$ & $1.4(1.0-2.0)$ & $1.4(1.0-1.9)$ & $1.4(1.0-1.9)$ \\
\hline $25-49$ & $19 / 93$ & $1.2(0.7-2.1)$ & I.I (0.7-I.9) & I.I (0.7-1.9) \\
\hline $50+$ & $13 / 53$ & $1.5(0.8-2.8)$ & $1.5(0.8-3.0)$ & $1.5(0.8-3.0)$ \\
\hline Past use & $84 / 422$ & $1.2(0.9-1.6)$ & $1.2(0.9-1.6)$ & $1.2(0.9-1.5)$ \\
\hline $1-6$ & $46 / 185$ & $1.5(1.1-2.1)$ & $1.6(1.1-2.2)$ & $1.5(1.1-2.2)$ \\
\hline $7-24$ & $27 / 177$ & $0.9(0.6-1.4)$ & $0.9(0.6-1.4)$ & $0.9(0.6-1.4)$ \\
\hline $25+$ & $11 / 60$ & I.I (0.6-2.I) & $0.9(0.5-1.9)$ & $0.9(0.5-1.9)$ \\
\hline \multicolumn{5}{|c|}{ Recency and Cumulative Dose of Glucocorticoid Prescriptions } \\
\hline Unexposed & $296 / 1763$ & 1.0 (reference) & I.0 (reference) & I.0 (reference) \\
\hline Current use & $670 / 1992$ & $2.1(1.8-2.5)$ & $1.9(1.6-2.3)$ & $1.9(1.6-2.3)$ \\
\hline I-999 & $116 / 281$ & $2.5(2.0-3.3)$ & $2.6(2.0-3.5)$ & $2.6(2.0-3.4)$ \\
\hline $1000-9999$ & $265 / 796$ & $2.1(1.7-2.5)$ & $1.9(1.6-2.4)$ & $1.9(1.6-2.3)$ \\
\hline $10,000+$ & $289 / 915$ & $2.0(1.7-2.4)$ & $1.7(1.4-2.1)$ & $1.7(1.4-2.1)$ \\
\hline Recent use & $118 / 495$ & $1.4(1.1-1.8)$ & $1.4(1.1-1.8)$ & $1.4(1.1-1.8)$ \\
\hline I-999 & $27 / 110$ & $1.5(1.0-2.3)$ & $1.6(1.0-2.5)$ & $1.6(1.0-2.5)$ \\
\hline 1000-9999 & $65 / 256$ & $1.5(1.1-2.0)$ & $1.4(1.1-2.0)$ & $1.4(1.1-2.0)$ \\
\hline $10,000+$ & $26 / 129$ & $1.2(0.8-1.9)$ & $1.2(0.8-1.9)$ & $1.2(0.8-1.9)$ \\
\hline Past use & $84 / 422$ & $1.2(0.9-1.6)$ & $1.2(0.9-1.6)$ & $1.2(0.9-1.5)$ \\
\hline I-999 & $37 / 146$ & $1.5(1.0-2.2)$ & $1.6(1.1-2.4)$ & $1.6(1.1-2.3)$ \\
\hline 1000-9999 & $30 / 190$ & $1.0(0.6-1.4)$ & $0.9(0.6-1.4)$ & $0.9(0.6-1.4)$ \\
\hline $10,000+$ & $17 / 86$ & I.I $(0.7-2.0)$ & I.I (0.6-I.9) & I.I (0.6-I.9) \\
\hline
\end{tabular}

Notes: anadjusted. ${ }^{b}$ Adjusted for phlebitis, COPD, diabetes, BMI, smoking. Conditional on the matching factors. ${ }^{\mathrm{C} A d j u s t e d}$ for phlebitis, COPD, diabetes, BMI, smoking, hormone replacement/OCP, epilepsy, alcohol. Conditional on the matching factors. Unexposed = glucocorticoid use I year or more prior to the index date. Current use = glucocorticoid use 30 days or less prior to the index date; Recent use = glucocorticoid use 90 days-less than 180 days prior to the index date; Past use = glucocorticoid use 180 days-less than I year prior to the index date.

Abbreviations: OR, odds ratio; $95 \% \mathrm{Cl}, 95 \%$ confidence interval. 
Table 5 Crude and Adjusted Odds Ratios for the Association Between Glucocorticoid Exposure and Venous Thromboembolism by Timing of Use and Route of Administration: Asthma Patients Aged 20-59 Years in CPRD GOLD 1995-2015

\begin{tabular}{|c|c|c|c|c|}
\hline \multicolumn{5}{|c|}{ Odds Ratio (95\% Confidence Interval) for Venous Thromboembolism (VTE) } \\
\hline & $\begin{array}{l}\text { Cases/ } \\
\text { Controls }\end{array}$ & Model I ${ }^{\mathrm{a}}$ OR $(95 \% \mathrm{Cl})$ & Model $2^{\mathrm{b}}$ OR $(95 \% \mathrm{Cl})$ & Model $3^{c}$ OR $(95 \% \mathrm{Cl})$ \\
\hline \multicolumn{5}{|c|}{$\begin{array}{l}\text { Recency and Route of } \\
\text { Glucocorticoid Prescriptions }\end{array}$} \\
\hline Unexposed & $296 / 1763$ & I.0 (reference) & I.0 (reference) & I.0 (reference) \\
\hline Systemic & $|7| / 28 \mid$ & $3.8(3.0-4.8)$ & $3.5(2.7-4.5)$ & $3.5(2.7-4.5)$ \\
\hline Inhaled & $701 / 2628$ & $1.6(1.4-1.9)$ & $1.5(1.3-1.8)$ & $1.5(1.3-1.8)$ \\
\hline Current Use & $670 / 1992$ & $2.1(1.8-2.5)$ & $1.9(1.6-2.3)$ & $1.9(1.6-2.3)$ \\
\hline Systemic & $142 / 177$ & $5.2(4.0-6.8)$ & $4.9(3.7-6.5)$ & $4.8(3.7-6.4)$ \\
\hline Inhaled & $528 / 1815$ & $1.8(1.6-2.2)$ & $1.7(1.4-2.0)$ & $1.7(1.4-2.0)$ \\
\hline Recent Use & $118 / 495$ & $1.4(1.1-1.8)$ & $1.4(1.1-1.8)$ & $1.4(1.1-1.8)$ \\
\hline Systemic & $|4 / 5|$ & $1.7(0.9-3.1)$ & $1.6(0.8-2.9)$ & $1.6(0.8-2.9)$ \\
\hline Inhaled & $104 / 444$ & $1.4(1.1-1.8)$ & $1.4(1.1-1.8)$ & $1.4(1.1-1.8)$ \\
\hline Past Use & $84 / 422$ & $1.2(0.9-1.6)$ & $1.2(0.9-1.6)$ & $1.2(0.9-1.5)$ \\
\hline Systemic & $15 / 53$ & $1.8(1.0-3.2)$ & $1.6(0.9-3.0)$ & $1.7(0.9-3.0)$ \\
\hline Inhaled & $69 / 369$ & I.I $(0.8-1.5)$ & I.I $(0.8-1.5)$ & I.I $(0.8-1.5)$ \\
\hline
\end{tabular}

Notes: 'Unadjusted. 'bdjusted for phlebitis, COPD, diabetes, BMI, smoking. Conditional on the matching factors. 'Adjusted for phlebitis, COPD, diabetes, BMI, smoking, hormone replacement/OCP, epilepsy, alcohol. Conditional on the matching factors. Unexposed = glucocorticoid use I year or more prior to the index date; Current use = glucocorticoid use 30 days or less prior to the index date; Recent use $=$ glucocorticoid use 90 days-less than 180 days prior to the index date; Past use $=$ glucocorticoid use I 80 days-less than I year prior to the index date.

Abbreviations: $\mathrm{OR}$, odds ratio; $95 \% \mathrm{Cl}, 95 \%$ confidence interval.

compared to 40-59 for current and recent use and for systemic use (Suppl 2). There was no effect measure modification when we stratified by BMI (data not shown).

Effect estimates were not materially different when we conducted sensitivity analyses restricted to patients who did not receive hormone replacement therapy or oral contraceptives in the 90 days before the index date $(\mathrm{n}=5335)$ (see Suppl 3), or to cases and their controls who were perfectly matched (Suppl 4).

\section{Discussion}

In this sample of asthma patients aged 20-59 years, we observed that current glucocorticoid use was associated with an increased risk of VTE, particularly in those with systemic use and prescriptions initiated within 6 months of the index date. The association remained statistically significant after adjustment for VTE risk factors, including age, sex (adjusted through matching), BMI, history of COPD, phlebitis, smoking, diabetes, hormone replacement therapy, and oral contraceptive pill use, though the effect diminished with increasing use. With a baseline asthma prevalence of 2.5/1000 people in this study, a twofold increase in the risk of VTE with glucocorticoid use would result in an additional 2.5 VTE cases/1000 asthma patients who initiate glucocorticoid.

Studies have demonstrated increased risks of VTE in association with estrogen replacement therapy, ${ }^{4,5}$ with risks between 2 and 4 times higher among women on estrogens compared to unexposed women. ${ }^{26-29}$ Results have been mixed for testosterone, but some studies suggest a twofold higher risk of VTE in the first 6 months of treatment in men exposed to testosterone compared to unexposed men., ${ }^{6,30}$ Given the impact of steroids on the endocrine system, particularly on sex hormones, insulin resistance and obesity, steroids including glucocorticoids may also increase VTE risk.

The apparent acute effect of steroids in the development of VTE has been documented in other studies. Sneeboer et al conducted a case-control study using 
a study design similar to the current study and showed a fourfold increase in recurrent VTE risk with current use of oral corticosteroids (OR 3.74; 95\% CI 2.04-6.87) and reduced risk with past use (OR $0.46 ; 95 \%$ CI $0.28-0.74) .^{21}$ A study conducted in Denmark showed a twofold increase in VTE risk with systemic glucocorticoid use (OR: 2.31, 95\% CI: 2.18-2.45) and inhaled glucocorticoid use (OR: 2.17, 95\% CI: 1.72-2.86) compared to non-use. ${ }^{11}$ However, only new use of inhaled glucocorticoid was associated with an increased risk of VTE. Haddad et al found an OR of 1.54, (95\% CI: 1.45-1.64) for preoperative steroid use on post-operative thromboembolism using data from the American College of Surgeons National Surgical Quality Improvement Program (NSQIP). ${ }^{9}$ Lieber et al found a higher risk of PE and DVT in neurosurgical patients exposed to at least 10 days of corticosteroid use $(\mathrm{OR}=1.47,95 \% \mathrm{CI}: 1.13-1.90)$ and $(\mathrm{OR}=1.55$, 95\% CI: $1.28-1.87)$ respectively. ${ }^{8}$ Similar to our study, Huerta et al found that current oral corticosteroid use was associated with the risk of VTE (OR: 3.05, 95\% CI: 2.51-3.69). ${ }^{14}$ The risk persisted with extended treatment, but the effect estimates gradually decreased with longer duration of use. Using a self-controlled design, Orsi et al showed a fourfold increase in VTE risk (HR: 3.51, 95\% CI: 2.55-4.80) during oral glucocorticoid treatment compared to unexposed periods. ${ }^{18}$ The risk increased in the week after treatment initiation and then gradually decreased over time. In our study, $>99 \%$ of systemic glucocorticoid use was oral, and we found a fourfold increase in VTE risk among current systemic glucocorticoid use compared to the non-exposed. When we assessed the effect of duration of use using the number of prescriptions received, we also found a gradual decrease in the effect estimate with longer duration.

Some investigators have questioned whether the association between glucocorticoid use and VTE can be explained by the underlying disease condition. ${ }^{20}$ Majoor et al showed a higher incidence of pulmonary embolism in severe asthma patients than mild-to-moderate patients and concluded that oral corticosteroid use and severe asthma were independent risk factors for pulmonary embolism (HR: $2.82 \quad(1.09-7.30)$ and $3.33 \quad$ (1.16-9.93), respectively). ${ }^{31}$ Restricting our study to asthma patients only allowed us to control for asthma and to evaluate the independent effect of glucocorticoid use on the risk of VTE.

In this study, for current exposure, the association with VTE was greater in the 20-39 age group than the 40-59 age group. This may be explained by the lower background risk of VTE in younger patients compared to older patients. The ORs of VTE were higher in females than males. In both sexes, the strongest effect was among current users which supports a causal association. However, the effect was stronger in females compared with males (aORs 2.3 versus 1.5 for any current use and 3.9 versus 2.9 in current users of systemic glucocorticoids). These findings were not explained by use of oral contraceptives or hormone replacement therapy. There was no evidence of effect measure modification by obesity (data not shown).

This study had several strengths. By limiting our study to a homogenous population comprising asthma patients without strong cardiovascular risk factors and by using an active comparator, we limited the potential for confounding by indication. We used a nested matched case-control design for this study to efficiently control important confounding factors. The CPRD data provided detailed information on exposure, dose, frequency, and type of prescription, which made it possible to assess exposure in several ways.

We attempted to control for confounding by indication in this study, but the possibility of residual confounding by severity of asthma exists. Severe asthmatics are more likely to be prescribed oral glucocorticoids, while mild asthmatics are more likely to be on intranasal or inhaled glucocorticoids, and the risk of VTE might be higher with severe asthma than mild asthma. We explored this by assessing different proxies for asthma severity, including dose and number of prescriptions. Our analyses showed a higher risk of VTE with systemic glucocorticoids use, however, dose and number of prescriptions were inversely associated with VTE risk.

This study has some limitations. There is potential for exposure misclassification in this study, but we do not expect it to be differential between cases and controls since the CPRD data are recorded prospectively without knowledge of future outcomes. Therefore, any exposure misclassification is more likely to bias the effect estimate towards the null and would not explain these results. Exposure misclassification could differ by severity of asthma and by the number of recorded prescriptions. Patients with severe disease and multiple prescriptions are more likely to have filled and used the drug, compared to patients with only a few prescriptions. Thus, patients with few prescriptions are more likely to be misclassified. Our results show that current glucocorticoid users with 1- 
6 prescriptions had a threefold increase in the odds of VTE (aOR: 2.9, 95\% CI: 2.2-3.7) (Table 4). If there were misclassification, the effect estimate would be higher which does not change our interpretation of the acute drug effect with glucocorticoid use.

Outcome misclassification of PE is unlikely since documented PE diagnosis is expected to be objective. However, outcome misclassification is still a possibility, especially for DVTs. To address this concern, we included receipt of anticoagulation in the case definition to validate the diagnosis. By conducting a case-control study with a well-defined outcome (VTE identified using Read codes as well as anticoagulant prescription codes) we attempted to identify true VTE cases and limit misclassification. Still, excluding cases who did not have an anticoagulant prescription may have resulted in the exclusion of fatal VTE and limited our cases to only milder VTE patients, which could impact the generalizability of our findings.

In summary, in this study population of 20-59-year-old asthma patients, we found evidence of an association between glucocorticoid use and VTE, particularly in new users of systemic glucocorticoids. Carefully weighing risk and benefit prior to initiating glucocorticoids in patients with asthma and monitoring for adverse events may be prudent. Further research to evaluate this association is warranted.

\section{Disclosure}

Dr David D McManus reports grants, personal fees from Bristol Myers Squibb, Pfizer, Flexcon, personal fees from Avania and Fitbit, non-financial support from Apple, grants from Boehringer Ingelheim, outside the submitted work. The authors report no other conflicts of interest in this work.

\section{References}

1. American Lung Association. Trends in Asthma Morbidity and Mortality; 2012.

2. von Eckardstein A, Wu FCW. Testosterone and atherosclerosis. Growth Horm IGF Res. 2003;13:S72-S84. doi:10.1016/S10966374(03)00059-5

3. Kapoor D, Malkin CJ, Channer KS, Jones TH. Androgens, insulin resistance and vascular disease in men. Clin Endocrinol (Oxf). 2005. doi:10.1111/j.1365-2265.2005.02299.x

4. Layton JB, Meier CR, Sharpless JL, Stürmer T, Jick SS, Brookhart MA. Comparative safety of testosterone dosage forms. JAMA Intern Med. 2015;175(7):1187-1196. doi:10.1001/ jamainternmed.2015.1573

5. Drake L, Hordinsky M, Fiedler V, et al. The effects of finasteride on scalp skin and serum androgen levels in men with androgenetic alopecia. J Am Acad Dermatol. 1999;41(4):550-554. doi:10.1016/ S0190-9622(99)80051-6
6. Houghton DE, Alsawas M, Barrioneuvo P, et al. Testosterone therapy and venous thromboembolism: a systematic review and meta-analysis. Thromb Res. 2018;172:94-103. doi:10.1016/j. thromres.2018.10.023

7. Stuijver DJF, Majoor CJ, Van Zaane B, et al. Use of oral glucocorticoids and the risk of pulmonary embolism: a population-based case-control study. Chest. 2013;143(5):1337-1342. doi:10.1378/ chest.12-1446

8. Lieber BA, Han J, Appelboom G, et al. Association of steroid use with deep venous thrombosis and pulmonary embolism in neurosurgical patients: a national database analysis. World Neurosurg. 2016;89:126-132. doi:10.1016/j.wneu.2016.01.033

9. Haddad A, Kantar R, Tamim H, Jamali FR, Taher A. Venous thromboembolism and preoperative steroid use, a retrospective cohort study from the ACS-NSQIP database: are surgical patients at risk? Blood. 2014;124(21):2865. doi:10.1182/blood.v124.21.2865.2865

10. Fowkes FJI, Price JF, Fowkes FGR. Incidence of diagnosed deep vein thrombosis in the general population: systematic review. Eur J Vasc Endovasc Surg. 2003;25(1):1-5. doi:10.1053/ejvs.2002.1778

11. Johannesdottir SA, Horváth-Puho E, Dekkers OM, et al. Use of glucocorticoids and risk of venous thromboembolism: a nationwide population-based case-control study. JAMA Intern Med. 2013;173 (9):743. doi:10.1001/jamainternmed.2013.122

12. Heit JA. The epidemiology of venous thromboembolism in the community: implications for prevention and management. In: The Vein Book; 2007. doi:10.1016/B978-012369515-4/50039-9

13. Weiss AJ, Elixhauser A. Overview of Hospital Stays in the United States, 2012. HCUP Statistical Brief \#180. October 2014. Agency for Healthcare Research and Quality, Rockville, MD. Available from: http://www.hcup-us.ahrq.gov/reports/statbriefs/sb180Hospitalizations-United-States-2012.pdf. Accessed January 05, 2022.

14. Huerta C, Johansson S, Wallander MA, García Rodríguez LA. Risk factors and short-term mortality of venous thromboembolism diagnosed in the primary care setting in the United Kingdom. Arch Intern Med. 2007;167(9):935. doi:10.1001/archinte.167.9.935

15. White RH, Keenan CR. Effects of race and ethnicity on the incidence of venous thromboembolism. Thromb Res. 2009;123:S11-S17. doi:10.1016/S0049-3848(09)70136-7

16. White RH. The epidemiology of venous thromboembolism. Circulation. 2003;107(23_suppl_1). doi:10.1161/01.CIR.0000078468.11849.66

17. Higgins PDR, Skup M, Mulani PM, Lin J, Chao J. Increased risk of venous thromboembolic events with corticosteroid vs biologic therapy for inflammatory bowel disease. Clin Gastroenterol Hepatol. 2015;13(2):316-321. doi:10.1016/j.cgh.2014.07.017

18. Orsi FA, Lijfering WM, Geersing G-J, et al. Glucocorticoid use and risk of first and recurrent venous thromboembolism: self-controlled case-series and cohort study. $B r \quad J$ Haematol. 2021;193 (6):1194-1202. doi:10.1111/bjh.17388

19. Waljee AK, Rogers MAM, Lin P, et al. Short term use of oral corticosteroids and related harms among adults in the United States: population based cohort study. BMJ. 2017;357:j1415. doi:10.1136/ bmj.j1415

20. Simion C, Campello E, Bensi E, et al. Use of glucocorticoids and risk of venous thromboembolism: a narrative review. Semin Thromb Hemost. 2021;47(6):654-661. doi:10.1055/s-0040-1722270

21. Sneeboer MMS, Hutten BA, Majoor CJ, Bel EHD, Kamphuisen PW. Oral and inhaled corticosteroid use and risk of recurrent pulmonary embolism. Thromb Res. 2016;140:46-50. doi:10.1016/j. thromres.2016.02.010

22. García Rodríguez LA, Pérez Gutthann S. Use of the UK general practice research database for pharmacoepidemiology. Br J Clin Pharmacol. 1998;45(5):419-425. doi:10.1046/j.1365-2125.1998.00701.x

23. Lawrenson R, Todd JC, Leydon GM, Williams TJ, Farmer RDT. Validation of the diagnosis of venous thromboembolism in general practice database studies. Br J Clin Pharmacol. 2000;49(6):591-596. doi:10.1046/j.1365-2125.2000.00199.x 
24. Goldhaber SZ. Risk factors for venous thromboembolism. J Am Coll Cardiol. 2010;56(1):1-7. doi:10.1016/j.jacc.2010.01.057

25. Anderson FA, Spencer FA. Risk factors for venous thromboembolism. Circulation. 2003;107(23_suppl_1). doi:10.1161/ 01.CIR.0000078469.07362.E6

26. Canonico M, Oger E, Conard J, et al. Obesity and risk of venous thromboembolism among postmenopausal women: differential impact of hormone therapy by route of estrogen administration. The ESTHER Study. $J$ Thromb Haemost. 2006;4(6):1259-1265. doi:10.1111/j.1538-7836.2006.01933.x

27. Olié V, Canonico M, Scarabin PY. Risk of venous thrombosis with oral versus transdermal estrogen therapy among postmenopausal women. Curr Opin Hematol. 2010;17(5):457-463. doi:10.1097/ $\mathrm{MOH} .0 \mathrm{~b} 013 \mathrm{e} 32833 \mathrm{c} 07 \mathrm{bc}$
28. Simon T, De Jonage-Canonico MBY, Oger E, et al. Indicators of lifetime endogenous estrogen exposure and risk of venous thromboembolism. $J$ Thromb Haemost. 2006;4(1):71-76. doi:10.1111/j.1538-7836.2005.01693.x

29. Bergendal A, Bremme K, Hedenmalm K, et al. Risk factors for venous thromboembolism in pre-and postmenopausal women. Thromb Res. 2012;130(4):596-601. doi:10.1016/j.thromres.2012.05.024

30. Martinez C, Suissa S, Rietbrock S, et al. Testosterone treatment and risk of venous thromboembolism: population based case-control study. BMJ. 2016:i5968. doi:10.1136/bmj.i5968

31. Majoor CJ, Kamphuisen PW, Zwinderman AH, et al. Risk of deep vein thrombosis and pulmonary embolism in asthma. Eur Respir $J$. 2013;42(3):655-661. doi:10.1183/09031936.00150312

\section{Publish your work in this journal}

Clinical Epidemiology is an international, peer-reviewed, open access, online journal focusing on disease and drug epidemiology, identification of risk factors and screening procedures to develop optimal preventative initiatives and programs. Specific topics include: diagnosis, prognosis, treatment, screening, prevention, risk factor modification,

Submit your manuscript here: https://www.dovepress.com/clinical-epidemiology-journal systematic reviews, risk \& safety of medical interventions, epidemiology \& biostatistical methods, and evaluation of guidelines, translational medicine, health policies \& economic evaluations. The manuscript management system is completely online and includes a very quick and fair peer-review system, which is all easy to use. 